\title{
Open access - A never ending story?*
}

\author{
Arne K. Richter ${ }^{* *}$ \\ European Geosciences Union (EGU), Germany
}

\section{Introduction}

Like all good stories, also the story of "open access" is occasionally rather confusing, and in which direction the story will develop and where and how it will end will depend on the point of view and the position of the person actually telling the story. Thereby, one has to remember that the story of "open access" is a trilogy rather than a monograph, since at least three sides with their own, independent ideas are involved: the subscribers (readers and researchers), the authors and the publishers.

The present report is therefore based on our own experience as scientists serving as readers, researchers, authors, editors, referees and officers for the European Geosciences Union (egu.eu) and its open access publications, with our open access publisher Copernicus Publications (copernicus.org), and with the software house for open access publishing Copernicus Systems + Technology (copernicussystems.net).

After a short retrospect on the history of the open access mission, we will discuss the advantages as well as the stumbling blocks of open access for researchers, authors and publishers and possible business models for open access publishing.

\section{Retrospect on the open access mission}

Only a few years ago the academic community was facing the following, rather hopeless situation:

- A steady increase in the subscription rates of academic work by about 250\% from 1983-2003 or of about $13 \%$ per year (journalprices.com) on the one hand, and, at the same time, a substantial decrease in the financial support of academic work in general on the other hand.

- A substantial surplus for commercial publishers from academic publications on the one hand, and a substantial transfer of "value adding" work from the publishers back to the academic community serving as authors, editors and referees free of charge anyhow.

- An explosive expansion of the internet and its accessibility worldwide and of academic work available on the internet, even though only toll-free.

Based on this situation, the academic community reacted in different ways:

\footnotetext{
*Lecture presented at the International Conference on Academic Publishing in Europe 2008, Berlin, Germany, 22-23 January, 2008, www.ape2008.eu. Published under the creative commons attribution licence.

** Address for correspondence: Dr. Arne K. Richter, Executive Secretary, European Geosciences Union, Buchenweg 30, D-37191 Katlenburg-Lindau, Germany. Tel.: +49 5552 7109; Mobile: +49 170 9078220; E-mail: arne.richter@egu.eu.
} 
- To require "Free Access to all Academic Work on the Internet" like the free access to all the other work presented on the web.

- Unrefereed, self-publishing of own work in the old fashion pre-print style (arXiv.org).

- Self-archiving of copies of published work (sherpa.ac.uk/romeo) or its archiving in public repositories and digital libraries (opendoar.org) for free public use.

- Cancellation of subscriptions and/or passover of entire editorial boards from expensive to less expensive journals and/or notices of termination from the position of editor and/or referee by individual scientists.

The request for "free access" was later on phrased more precisely as "open access" in the Bethesda Statement (2002) and in the Berlin Declaration (2003) in the following way:

- Barrier-free access to all academic work on the internet.

- License to copy, distribute, transmit and display the work publicly and to make and to distribute any derivative works to proper attribution of authorship (creative commons attribution license).

- Deposit the work and any supplemental material in at least one online repository for open access and long term archiving.

A survey by the Deutsche Forschungsgemeinschaft (DFG) on the other hand showed that the scientific community prefers to publish its work in proper, thematic scientific journals of high reputation and international, worldwide distribution, which guarantee a fair but rigorous peer review process of all academic work prior to publication, and which are included in the Citation Index with reasonable Impact Factors.

This survey clearly disapproves the preprint and self-archiving philosophies. Moreover, the distribution of author-generated copies of published work will be questioned with regard to its originality, and in the long term it will undermine the availability of genuin publications. And, finally, cancellations of subscriptions will most of all hurt the scientists themselves as readers and researchers but also as authors (I cannot read but my work is not read either).

Thus, the early messages of the open access mission are:

1. From readers and researchers: free access to and free downloads for derivative works of academic work of high quality.

2. From authors: publication in refereed open access journals of high reputation and international, worldwide distribution.

3. And from scientists in general: both services, in principle, at no cost, since scientists that cannot pay expensive subscriptions can also not pay expensive page/article charges.

The open access mission must therefore find a way which is a win-win situation for all three sides: for the scientists as readers and authors as well as for the open access publishers.

\section{Advantages of open access for subscribers and publishers}

Here "Open Access" benefits, first of all, from the explosive development and availability of a barrierfree internet worldwide, tending to become the only platform for any digital information and any digital work.

In addition, all electronic media being able to store digital work for purposes of information and/or for performing derivative works are steadily decreasing in price but increasing in its capacities: besides 
the PCs, Laptops and Palmtops, we will shortly have the TG stick, TG flash memories and fusel cells for powerful mini-workstations, as well as digital paper in user-friendly sizes. Moreover, more and more software, which is needed to perform professional original and/or derivative work, can be downloaded from the internet free of charge.

In parallel, the academic institutions and organizations have established a worldwide network of open access data bases, repositories, digital libraries and archives for open access academic work. This also includes data bases and archives for well defined topics, such as e.g. ADS, CAS, CSA, GeoRef etc., as well as the first long-term digital archive PORTICO of the US Library of Congress. Moreover, Alert Services, RSS feeds and DOI are active tools to inform the scientists online about the latest work of interest or its location on the internet.

Several organizations are working on the problem to improve the internet search-engines, such as Google or Google Scholar, to become more personalized and sophisticated with regard to the actual search as well as with regard to the personalized listings of the results (Get-What-You-Really-Want Search Engines). Another feature describing the quality of an open access publication could be the comparison of its content with the content of similar open access publications with regard to plagiarism and IPR (Intellectual Property Rights) violations to classify also the originality of a publication. Certainly, only academic work which is fully open accessible will profit from such advantages.

ISI - Thompson will also include quality, open access and purely digital journals in the Citation Index; and it has already been shown that the higher the percentage of open access publications in a given journal is the higher its Impact Factor.

A further important advantage for researchers is that open access work is published under the creative common attribution license, i.e. that any open access work may be copied, distributed, transmitted and displayed publicly and may be used to make and to distribute any derivative works to proper attribution of authorship without any annoying questions regarding copyright regulations any longer.

To help readers and researchers in studying open access literature online, certain open access publishers have started to change the format of their journals from portrait, double column (print) to landscape, on-screen publications with off-screen readable fonts (e.g. www.atmos-chem-phys-discuss.net).

Finally, the scientific community is more and more requesting that also the essential supplemental material of an original publication becomes publicly available, including reports, movies, numerical codes, experimental set-ups etc. Here only the internet with links to (self-)archived materials can serve as an answer.

In summary we may conclude:

- Open access - internet publishing provides the best overall services for the academic community for studies and derivative works, and

- Open access work is available free of charge worldwide.

And the message back to the publishers:

- Only quality, open access work will be cited and/or used for derivative works in future, i.e. non-open access work will not be read and therefore slowly forgotten within the scientific community.

- Publishers should use the numerous advantages of the free internet more rigorously, since typical tasks such as publishing, distribution and marketing of academic work as well as its storage and its problems with subscriptions, back issues, annual page budgets, investments in new publications etc. will not exist in their old fashion manner any longer in the open access world of publishing and will therefore represent a good chunk of expenses to be saved. 


\section{Stumbling blocks for open access}

Open access as the barrier- and subscription-free way of publishing academic work may be advantageous for readers and researchers; however, it is less advantageous for authors and publishers.

In the open access world the sources of income for publishers reduce to just page/article charges to be paid by the author. Thus, in the open access world the philosophy of payment is inverted from "subscription-paid and author-free" to "subscription-free and author-pays". In turn, these charges are therefore, in general, rather high. According to the conversion and the hybrid models offered by academic publishers, the article charges amount to about EUR 1,500-2,100 per article, depended on the size of the publisher and whether only the subscription fees or the overall turnover is converted into article charges.

In the past the subscription fees have been paid by the libraries or directly by the organizations. Page or article charges, however, have to be paid by the authors or their departments or institutes. Very often these departments and institutes have no explicit account for "page or article charges".

"Active" departments and research institutes would pay presently less for their subscriptions than for their article charges, if the price per article would amount to the value given above.

Even the signatories of the Berlin Declaration continue to pay subscription fees or a similar amount for their own authors to publish open access and are therefore still bound to the overall increase in the payment for published academic work. And the amount provided by some organizations to authors that want to publish open access is only a fraction of the article charges mentioned above.

And according to our experience in the geosciences, the old model of "subscription and author free" seemed to work more "ethical" than the open access model, since we were always able to distribute our own "pre-prints as accepted for publication" or even our off-prints to our friends and their departments and institutes, whereas for most of our colleagues a payment of article charges of the amount mentioned above will be impossible.

Finally, all major organizations in Europe will continue to support the classical, commercial publishers in the one or the other way. During the transition phase from toll-access to open access the amount of money for open access will remain relatively small.

Thus, serious open access publishers serving an international, worldwide community must investigate the details of applying the internet and the modern, digital technologies in their publication strategies for reducing the article charges for authors and yet earn an income sufficient for completing their tasks and responsibilities in the way asked for by the academic community.

\section{Open access online publishing for authors and publishers}

Due to the steep cuts in their budgets, scientists have learned to use their computers, the associated software and the internet to compile and to edit the results of their research ready-for-publication (called camera-ready some years ago), and to submit or to upload them online by themselves. This includes not only simple text but also equations, tables, plots, figures, photographs, movies, sound tracks etc. In this way, they are also able to interact with server-aided macros and templates to produce their articles online in the respective journal style ready for publication on the internet. Moreover, in the open access world readers and researchers will loose their interest in elaborated and expensive formats and will prefer simple, author-generated pdf or HTML files, wikis or blogs. Thus, the actual production of articles will rest mainly in the hands of the authors and of intelligent servers, and the staff at the production offices of 
publishers will just serve as a kind of "supervisor and quality control" rather than performing typesetting, editing, lay-outing, formatting etc. as in previous times. Purely author-generated, high-quality online publications are already included in our discussion journals (e.g. www.atmos-chem-phys-discuss.net).

Once an article has "passed", the server generates automatically customized XML files of the article and sends them to the respective data bases, archives, repositories and libraries worldwide, and via the alert services and RSS feeds also to the individual customers. In this way the article is published immediately worldwide and becomes at once part of all search engines and catalogues practically free of charge for the publisher.

In this way, an author receives prompt publication, a gigantic large readership, highest impact factors, and, last but not least, the copyright remains with the author. And for the publisher the cumbersome printing and distribution and tracing of printed issues has passed, while print-on-demand allows to distribute printed material at any time. Here a substantial part of past expenses of publishers can again be saved.

Remains the more complicated and time consuming and therefore more expensive part in the publication procedure, the procedure of the actual peer review of scientific work prior to publication. Here we are applying two different models:

1. The online yet classical review based on 2-3 independent referee reports - anonymous or eponymous.

2. The "open online peer review plus public discussion" procedure.

Since in both cases the entire review process rests in the hands of the editors and referees, it entirely can be serviced and monitored by intelligent servers, reducing the actual work of the editorial support office of the publisher more or less to a monitoring support of the work of the authors, editors and referees. Thereby, the second procedure has shown to have the following additional advantages:

- The manuscripts submitted for publication are more carefully compiled and of higher standard, so that editors and authors have less work to do for the review.

- Referees' and public comments are open access and published alongside the original article. Thus, value is added to the work of the referees and editors and the reports are generally of greater value to the author.

- The actual work of the editorial support office is roughly cut in half.

- Manuscripts are more intensively check against plagiarism and IPR violations.

At any time, however, either the author, the editor or the publisher can decide which services have to be added by the publishing staff for a successful publication of the manuscript. This also holds for copy editing, which is performed online by professional staff and which has to be paid by the author as an extra service.

From our model of publication we may therefore conclude:

- The entire process of submission, registration, peer review, production, publication and distribution of academic work can be handled, in principle, by the authors, editors and referees in cooperation with intelligent servers together with the publisher providing just the overall management, monitoring and quality control, the costs of which are merged into one fixed flat-rate per article.

- In addition, the author pays for the services asked for from the editorial support and the production offices of the publisher, page by page. 
This service charge model is already applied by the EGU to its open access journals; however, not yet fully automated, since the articles in all main journals are still edited and formated by the staff in the production offices in the old fashion portrait, double column, print-on-paper style.

\section{Business models for open access}

Due to an increasing pressure on the part of the science community towards open access, academic publishers will be urged to become, sooner or later, open access publishers. In the transition phase, which is already going on, different business models are discussed in parallel:

1. The Hybrid Model: in a journal, which is not open access in general, an author may choose the option that his/her article is open access in accordance to the creative commons attribution license. For this service the author has to pay an extra article charge (Springer's Open Choice Model).

2. The Conversion Model: a smaller, well-defined community organized through a dozen or so institutes/organizations and using only a small number of journals for research and publication, arrange with the corresponding publishers that by payment of the regular subscription fees the journals become open access (the CERN et al. Model).

3. The Article Charge Model: articles are publish in accordance to the classical procedure but are open access and the charge per article is minimized but fixed for the journal in question (BiomedCentral, PloS, IOP - New Journal of Physics).

4. The Service Charge Model: the less service an author requires from the editorial support and the production and distribution office of the publisher, the less the price per page (European Geosciences Union).

According to the first model, an institute subscribing to journals but asking its authors to publish open choice, pays actually twice. The conversion model, on the other hand, puts the same fiscal burden on the formerly subscribing institute, even if other institutes will have free access to their work. Models 3 is a rigid model leading still to article charges of the amount of about EUR 1,500, which is too high for the majority of the worldwide geosciences community. Model 4 is an automated, adoptive and flexible model, and in view of the very fast development of the software on the internet and for the web, this model will lead eventually to very low flat rates. Our dream is even that the makers of OpenOffice, ADOBE etc. develop an Open Access Publishing Tool to further reduce the page and finally the article charges to values, which will then become affordable for all authors worldwide. The mission statement of the EGU for publications is: dedicated to the pursuit of excellence and free and universal accessibility and affordability of scientific publications in all areas of geosciences and planetary and solar system sciences for the benefit of the scientists worldwide.

\section{Reputation}

The essential work for a publication is performed by the scientists acting as author, editor, referee, subscriber, reader and researcher. The decision about the quality, acceptance and value for further promotion purposes, and thus of the reputation of a journal also rests in the hands of the scientific community, including some of the parts of reputation, such as excellent editors, strong and competent referees, a steady flow of excellent, up-to-date and timely work, diversity etc. 
Thus, learned societies and organizations with their devoted communities and their tradition to listen to their grass-roots members and to follow and to incorporate their feedback in their work will be able to build up publications of highest standards, reputation, and excellency under the flag of open access, and by applying the automated, service charge model even at low investment and maximum services for their members and their worldwide communities.

Some years ago, the EGU has started a new open access journal with an open peer review process and public discussions right in the middle of a group of traditional, high ranked atmospheric and environmental journals. After only 4 years this journal was the number 1 publication according to its Impact Factor. A later journal started with an Impact Factor of 2.13 right from the beginning!

\section{Summary}

Open access is a mission for modern, online publishing of academic work with great potential and advantages for all sides involved: the researchers (subscribers), the authors and the publishers.

Based on the continuous increase in the subscription fees by about $13 \%$ per year and the resulting decrease in the availability of quality publications in the academic libraries, the general opinion was voiced that at least the work on the internet should be open accessible, i.e. barrier-free accessible and freely usable for any derivative work to proper attribution of authorship. Certainly, such a mission guarantees maximum worldwide distribution and impact of academic work and a number of simplifications and savings with regard to the tasks and responsibilities of publishers.

On the other side, however, it causes new, even more severe problems which have to be solved: in a strictly open access world the sources of income for publishers would reduce to page or article charges to be paid by the authors. Converting the income from subscription fees or even the overall income to article charges, one would presently get fees of the order of EUR 1,500-2,500 or even more per article. For most of our scientists worldwide it will be absolutely impossible to pay such a high price. Even more, in the previous subscription model in which publishing was free for authors, the "richer" colleagues could distribute copies, preprints and off-prints of their work to their "poorer" colleagues. In the new open access world reading would be free but publishing practically impossible for most of our colleagues. Thus, as long as the number of genuin publications in journals of high reputation is one of the key measure for the quality of a scientist, open access so far would only favour the rich but not necessarily the best scientists. Even though the open access publishers are taking a 10\% margin of freeof-charge pages in their annual budget into account, this would not be sufficient to serve the worldwide community in many areas in the geosciences.

Thus, if we want to put the open access mission on sustainable grounds, we have to continue to make rigorously use of the internet and the new web 2.0, the freely available software and intelligent servers and to develop the "Automatic Online Publication and Distribution System" to further reduce the tasks and responsibilities of publishers with regard to submission, peer review, production, distribution and marketing of academic work to an absolute minimum, just providing monitoring and quality control of the processes involved and the overall management of a journal. In this way, we envisage that article fees can be reduced to affordable flat-rates covered, may-be, by interested sponsors.

In this way, "Open access" is indeed a story that, at least for the moment, has not yet come to its end: to the battle call "free to read for everyone" we have to add "and free to publish for everyone" if we want to serve our entire community in a fair way! 